enantiomeric STs are twisted $4^{\circ}$ away in the opposite (CCW) sense.

Several variations on the general shape of the self-assembling molecules were investigated, and the best results were found with asymmetric building blocks in which one arm of the ' $\mathrm{V}$ ' is longer than the other. Each of the design elements - molecular shape, incorporation of $\mathrm{Br}$ atoms, the $\operatorname{Ag}(111)$ surface - is important for the formation of the triangles.

These considerations contribute to error-correction processes that are essential for the low defect densities observed in a system as complex as these STs. For example, three-fold coordination is required to stabilize each node in the network and the relative orientation of the three building blocks must be precise and also in epitaxy with the underlying Ag surface atoms. Thus, each bonding node in the fractal network is precisely defined. The asymmetrical nature of the molecular building block also contributes in an interesting way to the error-correction ability of the system. If a molecule binds at a site in a growing ST with the 'wrong' end, the opposite end of the molecule will not be able to form a stable node. Such defects are eventually annealed out so that another building block in the correct orientation can slot into place.

Temperature control is an essential element of any self-assembly process and it is no different in this study. An excessively high temperature would disrupt nascent ordered ST domains, but too low a temperature would tolerate the persistence of defective nodes (which are relatively weakly bound as a consequence of the building block design). Wang, Gottfried, Wu and co-workers point out that they have found a temperature range (67-80 K) where the molecular building blocks have sufficient mobility on the surface to examine many different binding sites, but also note that the cooling rate of the sample is critical in optimizing the ST structures. A cooling rate of only $0.02 \mathrm{~K}$ per minute allows the molecules the opportunity to gradually reduce their diffusivity while stabilizing the binding units at each node (relative to $k T$ ); under these conditions the formation of an almost complete fourth-order ST (a portion of one corner was missing, but the remainder of the structure can be characterized as being defect-free) containing nearly 300 molecules was observed.

By tuning the design of the molecules and the experimental conditions at each step, the system has been optimized to give the desired structures. In analogy to the natural world, this tuning process represents an accelerated evolution to produce an optimal structure and/or functionality, and this is often achieved through close interdisciplinary collaboration. Such an approach to surface-based self-assembly has been addressed in research reports ${ }^{6}$ and is an on-going discussion topic at international symposia, including 'Supramolecular Self-assembly at Surfaces' at the American Chemical Society meeting in March 2014 and the upcoming symposium of the same title at Pacifichem 2015. Design of complex systems (creating opportunities), discovery of novel properties (recognizing opportunities), and their development and tuning into multifunctional systems (realizing opportunities) are all essential aspects in advancing the field of complex self-assembled materials.

For the field of supramolecular chemistry - particularly for those researchers interested in surface functionalization and the realization of mathematical fractal designs - it is exciting to see the elements of rational instructed building-block design, kinetic control, and error correction combined in harmony to produce such beautiful structures. As we look to the next challenges in the design of complex molecular systems and their use in novel materials, it is valuable to consider the importance of these particular elements and the principles for tuning molecular systems to achieve them. The final piece of the puzzle is to put them together to achieve spontaneous self-assembly in synthetic systems that approaches the elegance and complexity of self-assembled structures that we see all around us in the natural world.

Steven L. Tait is in the Department of Chemistry, Indiana University, Bloomington, Indiana 47405, USA. e-mail:tait@indiana.edu

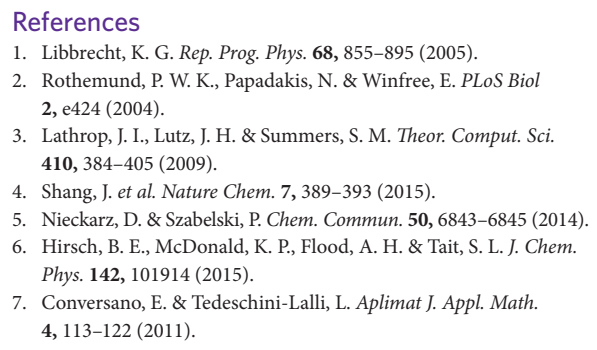

\section{References}

1. Libbrecht, K. G. Rep. Prog. Phys. 68, 855-895 (2005).

W. K., Papadakis, N. \& Winfree, E. PLoS Biol

410, 384-405 (2009)

Shang, J. et al. Nature Chem. 7, 389-393 (2

3-6845 (2014) Phys. 142, 101914 (2015) 4, 113-122 (2011)

\title{
Chromatin chemistry goes cellular
}

Analysing post-translational modifications of histone proteins as they occur within chromatin is challenging due to their large number and chemical diversity. A major step forward has now been achieved by using split intein chemistry to engineer functionalized histones within cells.

\section{Wolfgang Fischle, Dirk Schwarzer and Henning D. Mootz}

$\mathrm{H}$ istone proteins constitute the scaffold around which eukaryotic DNA is organized in the cell nucleus. These are targets of a plethora of post-translational modifications. Analysing the functional implications of the distinct patterns of histone modifications, also referred to as the histone code, is of high interest, as these control all aspects of genome function from transcriptional regulation to control of the cell cycle and repair of DNA damage.
Large mapping approaches are currently underway that aim to catalogue the distribution of histone modifications in relation to the genome in different biological conditions of cells. Such systems biology methodologies provide a bird's eye view of the complex signalling events that control chromatin behaviour thereby establishing functional correlations. However, in addition to these top-down mapping efforts (Fig. 1a, right) insights into the mechanistic function of histone modifications are required to understand this complex regulatory system.

Since post-translational modifications are not genetically encoded, their function must be deciphered on a biochemical level. Chemical biology approaches have provided experimental routes to specifically engineer modified histones in vitro, for instance by chemical modification of individual amino acid side chains ${ }^{1}$. Ligation of synthetic modified peptides and recombinant proteins 

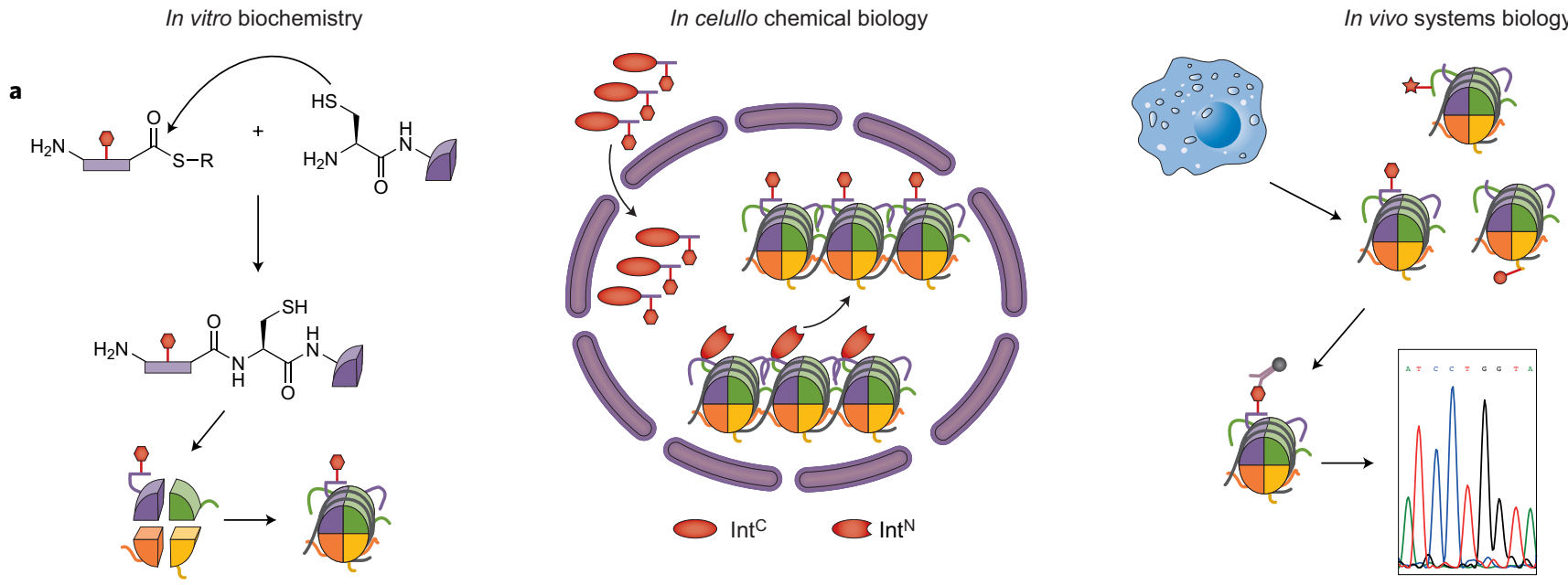

b

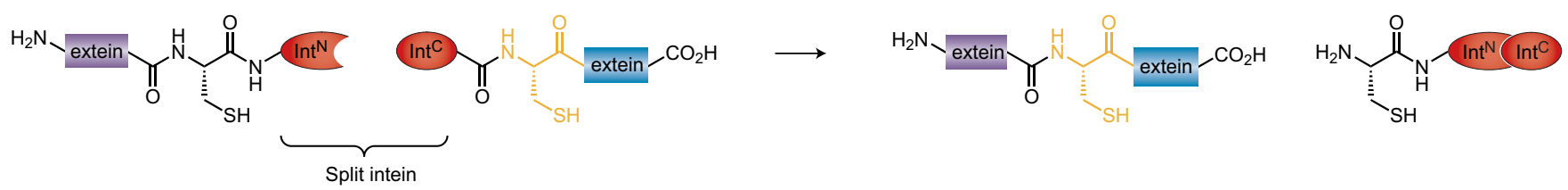

Figure 1 | Chemical biology of chromatin. a, Current approaches for studying the histone code. Left: Reconstituted nucleosomes containing modified histones generated by, for example, native chemical ligations are used to study chromatin regulation in vitro. Right: Correlations between global changes of histone modification patterns and alterations in gene expression are studied by chromatin immuno-precipitation/DNA-sequencing (ChIP-seq). Middle: Muir and co-workers report a new method for functionalizing histones under in cellulo conditions ${ }^{4}$. A peptide carrying a post-translational modification is linked to one part of a split intein $\left(\operatorname{Int} t^{C}\right)$. The target histone protein is fused to the other part of the split intein $\left(\operatorname{Int} t^{N}\right)$. b. Reaction scheme of protein trans-splicing.

through techniques such as native chemical ligation has been instrumental for creating modified histones, which are not attainable by classical means of protein production (Fig. 1a, left). Native chemical ligation was, for example, used to generate histone $\mathrm{H} 4$ acetylated on lysine 16 (ref. 2) and histone $\mathrm{H} 2 \mathrm{~B}$ ubiquitylated at its C-terminal tail $^{3}$, enabling seminal discoveries on the molecular consequences of these defined modifications. However, bottom-up approaches like that using reconstituted, modified chromatin are only able to dissect a limited functional context of the histone code. To bridge the large schism between in vivo systems biology and in vitro experimentation, new in cellulo chemical biology technologies are urgently needed.

In this issue of Nature Chemistry Muir and colleagues report a potentially versatile approach for the functionalization of histones in a cellular context based on protein trans-splicing (Fig. 1a, middle) ${ }^{4}$. The new experimental scheme enables targeting of the histones with small protein tags and fluorophores as well as introducing posttranslational modifications. Native chemical ligation is only poorly suited for reactions inside cells, because it requires high reagent concentrations, biolabile functional groups and harmful catalysts. Protein trans-splicing can be regarded as nature's equivalent of native chemical ligation and is mediated by the $\mathrm{N}$ - and C-terminal halves $\left(\operatorname{Int}^{\mathrm{N}}, \operatorname{Int}^{\mathrm{C}}\right)$ of a split intein protein (Fig. 1b). Upon their high-affinity association, Int ${ }^{\mathrm{N}}$ and Int ${ }^{\mathrm{C}}$ catalyse a remarkable rearrangement of the peptide backbone resulting in ligation of the flanking peptides (that is, exteins) and removal of the intein pieces. Importantly, $\operatorname{Int}^{\mathrm{N}}$ and $\mathrm{Int}^{\mathrm{C}}$ can be either chemically synthesized or genetically encoded and the reaction conditions of protein trans-splicing are amenable to cellular environments.

Muir and colleagues chemically synthesized an $\operatorname{Int}^{\mathrm{C}}$ (35 amino acids, aa) together with a short protein tag, a fluorophore or the C-terminal tail of histone $\mathrm{H} 2 \mathrm{~B}$ carrying the small protein ubiquitin (76 aa) as a special type of post-translational modification. The corresponding Int ${ }^{\mathrm{N}}$ was fused to the $\mathrm{C}$-terminus of the core of histone $\mathrm{H} 2 \mathrm{~B}$ on the genetic level and produced by expression in a human cell line. Despite the relative large size of $\mathrm{Int}^{\mathrm{N}}$ $(\sim 100 \mathrm{aa})$ in comparison with histone $\mathrm{H} 2 \mathrm{~B}$ (126 aa), the hybrid protein was incorporated into chromatin.
The cellular membrane represents an impermeable barrier for synthetic peptides like the $\mathrm{Int}^{\mathrm{C}}$ probes. The new work presents two different strategies for addressing this issue. The small $\mathrm{Int}^{\mathrm{C}}-\mathrm{H} 2 \mathrm{~B}$ probes (protein tag, fluorophore) were attached to a cellpenetrating peptide sequence (CPP). As CPPs mediate cellular uptake mostly via endocytosis, disulfide linkage was employed to ensure release of the cargo only in the reducing environment of the cytoplasm ${ }^{5}$. The efficiency of CPP-mediated transduction for larger cargos is, however, limited. Muir and colleagues bypassed this problem by applying the Int ${ }^{\mathrm{C}}-\mathrm{H} 2 \mathrm{~B}$-ubiquitin probe to isolated cell nuclei instead of intact cells. Owing to the permeability of the pore complexes, such nuclei can be supplemented with cofactors for enzymatic reactions, chemical compounds and larger proteins. Following cellular uptake or nuclear delivery, the $\mathrm{Int}^{\mathrm{N}}$ and $\mathrm{Int}^{\mathrm{C}}$ fragments associated and the peptide tag, fluorophore or the C-terminal tail of $\mathrm{H} 2 \mathrm{~B}$ carrying ubiquitin was transferred to the chromatin embedded $\mathrm{H} 2 \mathrm{~B}$ core fragment as desired.

The new method provides high versatility, as there is no apparent limitation to the structure of the synthetic peptide fragment 
used for trans-splicing. Non-native analogue structures of post-translational modifications, like the non-hydrolysable ubiquitin used in this study, are accessible.

It needs to be noted, though, that the split intein approach is not fully traceless. A single cysteine will remain in the product and a few surrounding amino acids will impact the splicing reaction.

The delivery of larger probes into cells remains a major obstacle. Here, future advancements will be key for a robust application of the technology. The use of isolated nuclei instead of intact cells might constitute a reasonable compromise for functionalizing histone proteins in a chromatin context. It seems likely that isolated nuclei will not recapitulate all aspects of the fine-tuned chromatin regulation network, but the fact that euchromatin and heterochromatin could be distinguished by $\mathrm{H} 2 \mathrm{~B}$ trans-splicing efficiency and that ubiquitylated $\mathrm{H} 2 \mathrm{~B}$ triggered known downstream events is promising in this regard. Besides targeting the C-terminal end of $\mathrm{H} 2 \mathrm{~B}$, the new study provided preliminary evidence that the approach is generally applicable to the $\mathrm{N}$-terminal tails of histones as well. These regions contain the highest density of posttranslational modifications. However, the size of the Int ${ }^{\mathrm{N}}$ half $(\sim 100 \mathrm{aa})$ needed for such reactions complicates its chemical synthesis and delivery into cells. Recently reported split inteins with very short $\mathrm{N}$-terminal fragments represent a promising alternative ${ }^{6}$.

In contrast to $\mathrm{N}$ - and C-terminal tails of proteins, the application of protein trans-splicing for accessing internal positions of a protein will not be straightforward, because splitting of the targeted protein into two fragments prior to reconstitution is required. This manipulation might in many cases result in misfolding and degradation. As temporal control of the reactions is essential for resolving the biological consequences of an engineered histone, inteins splicing significantly faster than the one used by Muir and colleagues will be interesting to test in the new scheme ${ }^{7}$.

Although the individual experimental parts for the new, elegant technology have been available for quite some time, the current study demonstrates for the first time that installation of chemical modifications by protein trans-splicing can be used to address a challenging cell-biological problem. Amber-stop-codon suppression for sitespecific incorporation of modified amino acids or derivatives that can be converted into post-translationally modified sites provides another powerful new approach without the problem of getting a synthetic peptide across the cell membrane ${ }^{8,9}$. Recent work demonstrated functionalization of a histone in yeast cells with a photo-inducible crosslinker, thereby enabling in situ chromatin interaction mapping inside cells ${ }^{10}$ Empowered with the new tools chemical biologists will without doubt uncover many secrets of cellular chromatin.

Wolfgang Fischle ${ }^{1}$, Dirk Schwarzer ${ }^{2}$ and Henning D. Mootz $z^{3}$ are at ${ }^{1}$ Max Planck Institute for Biophysical Chemistry, 37077 Göttingen, Germany. ${ }^{2}$ Interfaculty Institute of Biochemistry, University of Tübingen, 72076 Tübingen, Germany. ${ }^{3}$ Institute of Biochemistry, University of Münster, 48149 Münster, Germany.

e-mail:wfischl@gwdg.de;

dirk.schwarzer@uni-tuebingen.de;

henning.mootz@uni-muenster.de

\section{References}

1. Simon, M. D. et al. Cell 128, 1003-1012 (2007).

2. Shogren-Knaak, M. et al. Science 311, 844-847 (2006).

3. McGinty, R. K., Kim, J., Chatterjee, C., Roeder, R. G. \& Muir, T. W. Nature 453, 812-816 (2008).

4. David, Y., Vila-Perelló, M., Verma, S. \& Muir, T. W. Nature Chem. 7, 394-402 (2015).

5. Giriat, I. \& Muir, T. W. J. Am. Chem. Soc. 125, 7180-7181 (2003)

6. Thiel, I. V., Volkmann, G., Pietrokovski, S. \& Mootz, H. D. Angew. Chem. Int. Ed. 53, 1306-1310 (2014).

7. Carvajal-Vallejos, P., Pallisse, R., Mootz, H. D. \& Schmidt, S. R. J. Biol. Chem. 287, 28686-28696 (2012).

8. Neumann, H. et al. Mol. Cell 36, 153-163 (2009).

9. Nguyen, D. P., Garcia Alai, M. M., Kapadnis, P. B., Neumann, H. \& Chin, J. W. J. Am. Chem. Soc. 131, 14194-14195 (2009). 10. Wilkins, B. J. et al. Science 343, 77-80 (2014).

\section{AROMATICITY}

\section{A light-switched yin and yang pair}

In 1972, Baird showed theoretically that the electron counting rule for aromaticity and antiaromaticity in the lowest $\pi \pi^{\star}$ triplet state is opposite to that in the electronic ground state. A pair of compounds that manifests this reversal in character has now been identified and characterized experimentally for the first time.

\section{Henrik Ottosson and K. Eszter Borbas}

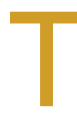
raditionally, aromaticity and antiaromaticity are described by Hückel's rule, which says that monocyclic fully $\pi$-conjugated hydrocarbons (annulenes) are aromatic if they have $4 n+2$ $\pi$-electrons and antiaromatic if they have $4 n$ $\pi$-electrons ${ }^{1}$. Yet, there are two ways that the electron count for (anti)aromaticity can be reversed so that $4 n \pi$-electrons correspond to aromaticity and $4 n+2 \pi$-electrons to antiaromaticity. First, the counting rule is reversed if the $\pi$-conjugated cycle is cut open and one end is rotated by $180^{\circ}$ before the cycle is reclosed into a Möbius band as described $^{2}$ by Heilbronner in a theoretical paper 1964, and with the first experiments reported in 2003 by Herges and co-workers ${ }^{3}$. The electron count is also reversed if one excites the annulene from its electronic ground state $\left(\mathrm{S}_{0}\right)$ to the first $\pi \pi^{*}$ triplet state $\left(\mathrm{T}_{1}\right)$ as shown ${ }^{4}$ theoretically by Baird in 1972. Numerous high-level quantum chemical studies have confirmed ${ }^{5,6}$ Baird's rule, but clear-cut experimental evidence for the reversal in (anti)aromaticity on excitation to $\mathrm{T}_{1}$ has, until now, been lacking.

So, how can you test experimentally for triplet state (anti)aromaticity? Aromatic compounds in the ground state have characteristic NMR chemical shifts, but the short lifetimes of triplet states compared with the NMR timescale renders this approach impossible. Moreover, paramagnetic molecules - such as molecules in their triplet states - are difficult to study by NMR spectroscopy because they give very broad signals. Instead, a way to implicitly confirm Baird's rule by experimental means is to find a pair of closely related compounds that are, respectively, aromatic and antiaromatic in the $\mathrm{S}_{0}$ state and whose experimental fingerprints are drastically different. When excited to the $\mathrm{T}_{1}$ state the (anti)aromatic character of the two compounds will, according to Baird's rule, reverse, and this should be reflected in their fingerprints, which then also interconvert. The pair of compounds should be a 'yin and yang' pair in the $S_{0}$ state and a 'yang and yin' pair in the $\mathrm{T}_{1}$ state. Writing in Nature Chemistry, Dongho Kim, Atsuhiro Osuka and co-workers now report ${ }^{7}$ on such a pair; the hexaphyrins $\mathbf{R} \mathbf{2 6 H}$ and $\mathbf{R 2 8 H}$ (Fig. 1). 